\title{
Retrograde flow in aortic isthmus - Trigger to deliver growth restricted fetuses between 30-34 weeks of gestation?
}

\author{
Shalaka Bansode ${ }^{1}$, Bijoy Balakrishnan ${ }^{1}$, Meenu Batra ${ }^{1}$, Sreeja P.S ${ }^{1}$, Swapneel N.Patil ${ }^{1}$, and \\ K.K Gopinathan ${ }^{1}$
}

${ }^{1}$ Edappal Hospitals Pvt Ltd

May 5, 2020

\begin{abstract}
OBJECTIVE: To evaluate the association of aortic isthmus (AoI) circulation assessed by doppler imaging in growth restricted fetuses with perinatal outcome. DESIGN: prospective longitudinal observational study. SETTING- Tertiary referral center POPULATION- Intrauterine growth restriction (IUGR) ( $\mathrm{n=38}$ ) between 24-36 weeks' gestation. METHODS- Doppler examination in aortic isthmus, umbilical artery (UA), middle cerebral artery(MCA), and ductus venosus(DV) was performed. The relation between antegrade $(\mathrm{n}=25)$ and retrograde flow $(\mathrm{n}=13)$ in AoI ,other Doppler parameters with perinatal outcome was analyzed. RESULTS: Retrograde AOI group had higher rates of adverse perinatal outcome (92.3\% vs. $72 \%)$ with $63.1 \%$ sensitivity and $87.5 \%$ specificity. Overall perinatal mortality (intrauterine death and neonatal death) was higher in retrograde group (23\%).No perinatal mortality in antegrade AoI group. Cases with absent end diastolic flow (AEDF) or reverse end diastolic flow (REDF) in the UA and retrograde flow in AoI and normal DV flow had $40 \%$ prenatal mortality as $25 \%$ in those with abnormal DV Doppler . CONCLUSION: Rretrograde blood flow in AoI is associated with adverse perinatal outcome, particularly intrauterine demise, neonatal death, RDS and increased duration of NICU stay. Even though the DV flow is normal adverse outcome might be suspected in fetuses with AEDF/REDF in UA and retrograde flow in AoI. Thus, retrograde flow in AoI might be considered as an additional trigger for delivering IUGR fetuses 30-34 weeks with AREDF in UA but requires further evaluation by large longitudinal studies . Keywords - Intrauterine growth restriction, doppler ultrasonography; fetal aortic isthmus; perinatal outcome.
\end{abstract}

\section{Main text}

Retrograde flow in aortic isthmus - Trigger to deliver growth restricted fetuses between 30-34 weeks of gestation?

Dr. Shalaka Bansode, Dr. Bijoy Balakrishnan, Dr.Meenu Batra, Dr.Sreeja P.S, Dr. Swapneel Patil, Dr. K.K.Gopinathan.

Department of Feto-maternal medicine, CIMAR, Edappal Hospitals Pvt Ltd, Edappal, Malappuram, Kerala. India 679576

\section{INTRODUCTION}

Intrauterine growth restriction (IUGR) due to placental insufficiency is predominantly a vascular disorder. It is associated with adverse short term and long term outcomes [1,2, and 3]. Fetal well being tests and indices could be roughly classified as chronic and acute. Chronic indices (abnormal doppler flow in umbilical artery(UA) or middle cerebral artery(MCA) become progressively abnormal due to increasing hypoxemia or hypoxia while acute indices(reversal in ductus venosus (DV) represent severe fetal hypoxia and metabolic acidosis and usually precede fetal death in few days. Doppler examination of the umbilical artery (UA) and the middle cerebral artery (MCA) have proved to be good predictors of adverse perinatal outcome. 
However, because the risks of iatrogenic prematurity are very high before 32 weeks' gestation, further doppler parameters are needed to assess fetal compromise. Venous Doppler patterns together with gestational age (GA) at delivery have been advocated as the best predictors of adverse perinatal outcome in IUGR fetuses ${ }^{[4]}$ however are too late and represent the failed fetal defence mechanism against hypoxia ${ }^{[5]}$. Thus there is a gap of few days to weeks between these early and late signs during which new parameters are needed to document the progression of fetal deterioration in more better and accurate way. This will help to identify those fetuses whose defence mechanism against severe hypoxia and acidemia are about to fail but yet not failed.

Aortic isthmus blood flow pattern reflects the balance between both ventricular outputs and the existence of differences in the impedance in either vascular system ${ }^{[6]}$. Its role as a short term marker of adverse perinatal outcome $^{[7]}$ and a long-term predictor of neurodevelopmental outcome ${ }^{[8,9]}$ have been proposed. Abnormal AoI impedance indices occur prior to cardiac decompensation ${ }^{[10]}$. Retrograde flow in aortic isthmus occurs commonly after the abnormal flow in the umbilical artery and before abnormal ductus venosus flow. Thus the aim of this study was to evaluate the association between AoI doppler changes and adverse perinatal outcome in growth-restricted fetuses and neurodevelopemental outcome between 2-4yrs of age and to find out whether can we use retrograde flow in aortic isthmus as an additional trigger to deliver growth restricted fetuses between 30-34 weeks of gestation where yet there is a dilemma between continuing the surveillance vs. delivering prematurely due to lack availability of clear indicators .

\section{MATERIAL AND METHODS:}

This prospective longitudinal observational study was conducted from January 2018 to February 2019 in a tertiary referral fetal-maternal medicine centre at the EDAPPAL HOSPITAL PVT LTD, EDAPPAL, MALAPPURAM, KERALA. All pregnant women visiting at CIMAR Out Patient Department with USG suggestive of IUGR fetus by Delphi consensus definition ${ }^{[11]}$ between 24-36 weeks of gestation and fulfilling the other inclusion criteria were included in the study.

Written informed consent was obtained from each patient enrolled into the study. The further ultrasonographic surveillance was done depending upon the USG findings. Intrauterine growth restriction was defined as per Delphi consensus [11].

Estimated fetal weight percentile was calculated by using Hadlock growth charts as standard. Inclusion criteria : Women willing to take part in the study, Singleton pregnancy,GA confirmed by USG in the first trimester, absence of structural malformations or chromosomal abnormality, women with USG findings satisfying above mentioned definition criteria of IUGR and last Doppler examination performed within $48 \mathrm{~h}$ before delivery.

Women not willing to take part in the study or multiple pregnancy or fetuses with chromosomal abnormalities or structural anomalies were excluded from the study.

\section{Ultrasound examination:}

Image-directed pulsed and colour Doppler equipment GE Voluson E8 and E6(General Electric Healthcare ,Europe) ultrasound machine with transabdominal transducer was used to perform Doppler studies of the Umbilical artery(UA), Middle cerebral artery(MCA),Ductus venosus ( DV) and aortic isthmus (AoI) .

Doppler indices were calculated from waveforms obtained during minimal fetal activity and the absence of fetal breathing movement, at an insonation angles $<30^{\circ}$. At least three Doppler waveforms were taken for each measurement and the most representative one was included for analysis. The PI was recorded for the Umbilical artery, Middle cerebral artery and Ductus venosus. The parameters were plotted against established centiles for gestation using Barcelona fetal medicine reference charts.

Umbilical artery Doppler and DV Doppler were assessed as PI value percentile or end-diastolic velocity was classified as absent (AEDF) or reversed (REDF). Umbilical artery with PI $>95^{\text {th }}$ centile for GA or with AREDF was considered as abnormal umbilical artery Doppler flow. MCA-PI $<5$ th centile for gestational 
age was considered as an evidence of cerebral redistribution or 'brain sparing 'and considered as abnormal MCA doppler flow. Also ratio of MCA PI and UA PI that is cerebroplacental ratio (CPR) was calculated. CPR $<2^{\text {nd }}$ percentile for GA was considered as abnormal. Similarly DV with PI $>95^{\text {th }}$ percentile for GA or with AREDF was considered as abnormal DV doppler flow.

\section{Aortic isthmus Doppler velocimetry:}

AoI Doppler was assessed in the cross-sectional 3VT view.

The 3VT view was obtained at the level of the fetal mediastinum by moving the transducer obliquely cephalad from the four-chamber view. At this point, the pulmonary trunk, ductus arteriosus, aortic arch, AoI and superior vena cava are clearly demonstrated, with the aortic and ductal arches forming a $\mathrm{V}$ configuration pointing to the posterior spine. The $\mathrm{V}$ shape shows the convergence of the AoI and the arterial duct, helping to identify where the range gate should be placed. Once the vascular segment identified, colourdirected pulsed-wave Doppler was adjusted to high velocities so that the great vessel's blood flow will be homogeneous in colour and will show no aliasing. The pulsed wave gate size was adjusted to avoid recording signals from the adjacent vessels. Velocity waveforms were recorded during fetal quiescence with the angle of insonation close to $0^{\circ}$ and no more than $30^{\circ}$.

The aortic isthmus waveform was assessed as presence of diastolic flow or absent or reversed diastolic flow (Figure $1 \mathrm{~A}$ and $1 \mathrm{~B}$ ). In cases of some reversal of diastolic flow but predominant antegrade flow, it was considered as antegrade flow while if predominant flow is retrograde then it was considered as retrograde flow in aortic isthmus. When there was no flow in the diastole was taken as absent diastolic flow in AoI.

Staging of IUGR- After growth assessment and doppler study, IUGR stage was assigned by using staging system given by Gratacos et al 2014 ${ }^{[12]}$. The Doppler parameters and impedance indices were plotted against established centiles for gestation using Barcelona fetal medicine reference charts.

Statistical analysis was performed using SPSS 23.0 Doppler variables and perinatal outcome were analyzed by Pearson's Chi-square or Fisher's exact test as indicated. The predictive value of the AoI and other Doppler variables in predicting adverse perinatal outcome was assessed by estimation of sensitivity, specificity, predictive values and likelihood ratios and by multiple regression analysis.

Delivery of IUGR fetuses was decided depending upon weeks of gestation and doppler flows and by an abnormal fetal heart rate trace (Table 1). Retrograde flow in aortic isthmus was not used as a trigger for delivering these fetuses.Mode of delivery was by emergency Caesarean section in these cases, irrespective of the stage of IUGR as a routine protocol of the institute .Protocol for follow up and delivery was followed as mentioned in above staging system.

\section{Outcome}

Primary outcome parameters included still birth, neonatal death,, development of respiratory distress, bronchopulmonary dysplasia, intraventricular hemorrhage, Transient tachypnea of newborn(TTN) ,Hyaline membrane disease (HMD), necrotizing enterocolitis(NEC), sepsis, and neonatal intensive care (NICU) stay longer than 7 days. Perinatal outcome was defined as adverse when any of these complications was present.

\section{RESULTS}

Optimal aortic isthmus waveform assessment could be obtained in all cases .Out of 45 cases recruited 2 cases lost to follow up and 5 cases got delivered at other hospital, in these cases aortic isthmus doppler analysis at least 48-72 hours prior to delivery was not available, hence these cases were excluded from the study. Of the total analyzed 38 cases, 25 had antegrade diastolic flow in the aortic isthmus and 13 had retrograde diastolic flow in the aortic isthmus.

\section{Demographic and obstetric data of the study population is mentioned in Table 2}

There was no significant difference in these two groups with respect to maternal age, type of conception and parity. There was high rate of preeclampsia in retrograde group (46\%) as compared to antegrade group 
(24\%). Gestational age at delivery $(\mathrm{p}=0.021)$ and birth weights $(\mathrm{p}=0.022)$ were significantly lower in the retrograde flow in the aortic isthmus group.

Distribution of complications in antegrade and retrograde flow in the aortic isthmus groups is given in Table 3 and figure 2.

Overall adverse perinatal outcome includes intrauterine death and postnatal complications. Retrograde AOI group had higher rates of adverse perinatal outcome (92.3\% vs. $72 \%)$. Only $1 / 13$ of retrograde group did not have any adverse perinatal outcome. There was 1 intrauterine death and 2 neonatal deaths in the study population .All these cases were from retrograde AOI group. Perinatal mortality was $3 / 13(23 \%)$ in retrograde flow in AoI group while there was no perinatal mortality in antegrade flow in AoI group.

Necrotizing enterocolitis was seen in one case from retrograde group. Respiratory distress syndrome was more common in retrograde AOI group $(70 \%$ vs. $20 \%)(\mathrm{p}=0.033)$. For remaining complications there was no such difference in these two groups. Distribution of complications in antegrade and retrograde flow in the aortic isthmus groups is shown in the figure. 4 below.

The relationship between aortic isthmus Doppler flow and other Doppler parameters was also assessed and is given in table 4 .

There was significant association of retrograde diastolic aortic isthmus flow with abnormal umbilical artery, abnormal MCA, and abnormal DVdoppler.12/13(92.3\%) cases of retrograde group had abnormal umbilical artery doppler flow (PI $>95^{\text {th }}$ centile or AREDF). Similarly 12/13(92.3\%) cases of retrograde group had abnormal MCA Doppler flow (PI $<2^{\text {nd }}$ centile). 5/13 case of retrograde group had abnormal DV doppler ( $>95^{\text {th }}$ centile). $5 / 7$ (71.4\%) of abnormal DV had reverse diastolic flow in the aortic isthmus.

The predictive value of the AoI and other Doppler parameters for an adverse perinatal outcome were also assessed.The predictive value of the AoI and other parameters for an adverse perinatal outcome is given in Table 5.

UA and AoI retrograde Doppler flow patterns and gestational age at delivery $<34$ weeks of gestation had the highest positive likelihood ratios. Abnormal AOI Doppler has $63 \%$ sensitivity, specificity of $87.5 \%$, a good positive predictive value (92.3\%) and positive likelihood ratio of (5) for the prediction of adverse perinatal outcome.

following equation of multiple regression analysis was used to see the relationship between Doppler variables and WOG with perinatal outcome -

Perinatal Outcome $=1.685-(0.134 \times \mathrm{UA})-(0.295 \mathrm{x} \mathrm{MCA})-(0.097 \times \mathrm{DV})+(0.164 \times \mathrm{xOI})+(0.047 \mathrm{x}$ WOG).

It stated that ,based on standardized coefficient, AOI (0.19) is the most important factor to extract perinatal outcome followed by WOG (0.047) .

Prematurity (delivery $<34$ weeks of gestation) alone has a sensitivity (70\%) and high specificity (93\%) and a high positive likelihood ratio (8.75) as a predictor of adverse perinatal outcome and overall mortality.

In the retrograde AOI group outcome was also assessed in two subgroups that is one with normal DV Doppler and another with abnormal DV Doppler (Table S1). No statistically significant difference was found in these two subgroups in terms of following parameters.

However, there was higher percentage of RDS (80\%vs $38 \%$ ) and NICU stay $>7$ days (100\%vs $50 \%$ ) in retrograde AoI group with abnormal DV Doppler. Also the only case with NEC had abnormal DVflow .Also perinatal mortality was higher $(25 \%)$ in retrograde AoI group with normal DV.

This subgroup analysis of retrograde AoI group shows that the perinatal outcome can be adverse even when the DV flow is normal especially in terms of perinatal mortality.

There were total 18 cases in which weeks of gestation at delivery was $<34$ weeks. 
Out of these majority (13) cases were delivered prematurely because of fetal indication that is abnormal Doppler and in only 5 cases maternal indication was present for delivery $<34$ weeks.

Analysis of the fetuses with AEDF or REDF in UA with retrograde AoI by sub grouping in those having normal DV and those with Abnormal DV was also done, shown in Table no. S2 .

It shows that, the group with normal DV did not require NICU admission for $>7$ days, while $\frac{3}{4}(75 \%)$ cases with abnormal DV required NICU stay $>7$ days. There was 1 IUD and 1 NND from the group with normal DV. while 1 NND from DV abnormal group. So, even with normal DV Doppler flow the perinatal mortality (40\%) was found in AEDF/REDF with retrograde AOI group with normal DV flow- P value $0.638,95 \%$ CI $(-0.454-0.754)$

Longitudinal changes in Doppler parameters in these IUGR fetuses are shown in figure 3 The median values for umbilical artery PI $>95^{\text {th }}$ centile, Absent end diastolic flow (AEDF), Reverse end diastolic flow (REDF) ,Middle cerebral artery PI $<5^{\text {th }}$ centile ,Retrograde flow in aortic isthmus and DV Pi $>95$ the centile were $8,1,0,7,2$ and 0.5 respectively .

\section{DISCUSSION :}

\section{Main Findings-}

There is a diversity and lack of uniform model which would reflect hemodynamic changes in pregnancy with IUGR. The surveillance of these IUGR cases and taking a clear cut decision whether to deliver these fetuses or not and if when, thus weighing the risk of intrauterine death by prolonging the gestation versus delivering the fetus prematurely is the major concern. Our data call into question whether can be use retrograde flow in aortic isthmus as a trigger to deliver these growth restricted fetuses.

This study supports the association of retrograde flow in the aortic isthmus with the adverse perinatal outcome as suggested by ${ }^{[13]}$ M.Del Rio et al and Hidar et al ${ }^{[14]}$. In our study, Retrograde AoI group had higher rates of adverse perinatal outcome (92.3\% vs. $72 \%)$. Abnormal AoI had sensitivity of $63.1 \%$, a high specificity $(87.5 \%)$ and a PPV (92.3\%) and likelihood ratio (5) for prediction of adverse perinatal outcome. Overall perinatal mortality (intrauterine death and neonatal death) was higher in retrograde group 3/13(23\%). There was no perinatal mortality in antegrade flow in AoI group. Thus suggesting a potential role for Doppler imaging of the AoI in the clinical surveillance of severe IUGR fetuses .

In our study, there was a highly significant association of retrograde diastolic aortic isthmus flow with abnormal umbilical artery (92.3\% vs. $36 \%)(\mathrm{p}=0.013)$ similar to study by Del rio etal ${ }^{[13]}$. Thus suggesting that retrograde diastolic flow in the AOI represents a group with more severe and earlier onset of placental insufficiency.

In a study by del rio et $\mathrm{al}^{[13]}$ and Makikallio $\mathrm{K}$ et $\mathrm{al}^{[15]}$, MCA vasodilatation did not differ between the antegrade and retrograde groups. However in our study, MCA vasodilatation differed in the antegrade and retrograde groups ( $48 \%$ vs. $92.3 \%$ ) respectively .

Similar to Del rio et al ${ }^{[13]}$, our study shown a strong association between the presence of abnormal DV flow and retrograde flow in the AoI. $(38 \%$ vs. $8 \%)(\mathrm{P}=0.034) .5 / 7(71.4 \%)$ of cases with abnormal DV flow had reverse diastolic flow in the AOI. This suggests well corelation of these parameters with the progressive deterioration of cardiac function occurring in IUGR. All these findings suggested significant corelation of retrograde flow in aortic isthmus with abnormal flow in UA,MCA and DV.

Del rio et al ${ }^{[13]}$ proposed that AoI PI and DV-PI independently predict adverse outcome. And in preterm growth restricted fetuses, AoI blood flow becomes abnormal on average 1 week before DV blood flow does. In our study ,retrograde flow in AoI was seen on an average 2 days before delivery and abnormal DV PI was seen 1 day before delivery .

We have also analyzed, the fetuses with AEDF or REDF in UA with retrograde AoI by sub grouping as, those having normal DV and those with Abnormal DV . There were 2 perinatal deaths from the group with 
normal DV while 1 NND from abnormal DV group . So , even with normal DV Doppler flow the perinatal mortality (40\%)was found in AEDF/REDF with retrograde AOI group. Thus even though the DV flow is normal adverse outcome might be suspected in fetuses with AEDF/REDF in UA and retrograde flow in AoI. Thus providing a better window for delivering the IUGR fetuses before failure of fetal compensatory mechanisms to hypoxia.

\section{Results -}

Our study confirms previous observations that retrograde blood flow in the AoI is associated with adverse perinatal outcome, particularly intrauterine demise ,neonatal death ,RDS. Retrograde flow in AoI correlates significantly with abnormal flow in Umbilical artery, Middle cerebral artery and Ductus venosus.

Abnormal aortic isthmus flow is generally seen prior to abnormal changes Ductus venosus Doppler .

\section{Clinical Implications -}

Even though the DV flow is normal adverse outcome might be suspected in fetuses with AEDF/REDF in UA and retrograde flow in AoI. Thus providing a better window for delivering the IUGR fetuses before failure of fetal compensatory mechanisms to hypoxia.

Thus retrograde flow in AoI might be considered as an additional trigger for delivering IUGR fetuses between 30-34 weeks with AREDF in UA.

Also , AoI Doppler might be considered as an additional clinical parameter in the routine assessment of hemodynamically compromised growth-restricted fetuses.

\section{Research Implications-}

Thus by considering above all findings we could suggest that ,Retrograde flow in AoI might be considered as an additional trigger for delivering IUGR fetuses between 30-34 weeks with AREDF in Umbilical artery and might be considered as an additional clinical parameter in the routine assessment of hemodynamic ally compromised growth-restricted fetuses.

Hence suggesting an opportunity to combine the Umbilical artery ,Aortic isthmus and DV Doppler flows in minimizing the adverse perinatal outcome which should be studied further ..

Gratacos et al ${ }^{[16]}$ suggested that before 26 weeks and after 28 weeks, gestational age alone is the strongest predictor of perinatal mortality in early-onset IUGR. However, in the group between 26 and 28 weeks of gestation, the DV may provide useful information and allow stratification between high and low risks of perinatal mortality. As by the staging classification given by Gratacos et al ${ }^{[12]}$,AEDF in umbilical artery and reversal in AoI suggests severe placental insufficiency while REDF in umbilical artery and abnormal DV PI suggest suspicion of fetal acidosis

Also suboptimal neurological development was found among children who had net reversed diastolic flow in the AoI antenatally ${ }^{[8]}$,Considering all this it might be better to consider AEDF/REDF in UA and reversal of flow in AoI as a sensible marker for delivering the IUGR fetus especially between 30-34 weeks of gestation before the fetal compensatory mechanisms to hypoxia fail .

\section{Strengths and Limitations-}

Prospective nature of the study and simplified subjective approach of AoI waveform analysis rather than objective assessment of waveform were the merits of our study.

This study has limitations like small sample size and prematurity .

Delivery $<34$ WOG had sensitivity $(70 \%)$,Specificity ( $92 \%$ ) and high positive likelihood ratio of 8.75 for prediction of adverse perinatal outcome, supporting the important role prematurity in the prediction of perinatal outcome .

And also retrograde flow in the aortic isthmus was not used as trigger for delivery . 


\section{Conclusion}

To conclude, this study confirms previous observations that retrograde blood flow in the AoI is associated with adverse perinatal outcome, particularly intrauterine demise, neonatal death ,RDS. Retrograde flow in AoI correlates significantly with abnormal flow in Umbilical artery,Middle cerebral artery and Ductus venosus. And even though the DV flow is normal adverse outcome might be suspected in fetuses with AEDF/REDF in UA and retrograde flow in AoI. Thus, retrograde flow in AoI might be considered as an additional trigger for delivering IUGR fetuses between 30-34 weeks with AREDF in Umbilical artery .However further large prospective studies and randomized controlled trials taking retrograde flow in aortic isthmus along with AREDF in UA as a trigger to deliver are required for evaluating immediate perinatal as well as long term neurological outcome. This might help to improve the surveillance protocols and perinatal as well as long term neurodevelopemental outcome in IUGR fetuses .

Disclosure of interests : The authors report no financial, personal, political, intellectual or religious conflict of interest

\section{Contribution to authorship:}

DR. SHALAKA ARUN BANSODE - Principal author

I being the Principal author has carried out this study after planning with my co authors and carried out the case collection ,study analysis and writing up of my work .

\section{Co-authors}

DR.BIJOY BALAKRISHNAN - He being the guide and Head of Fetomaternal unit at ,Edappal hospital had the main role in the concept and planning of this study and helped in the analyzing and writing of this work

DR.MEENU BATRA - Role in the concept and planning of this study and helped in the writing of this work

DR.SREEJA P.S - Role in the planning of this study and helped in the carrying out this work .

DR.SWAPNEEL .N.PATIL- Role in the planning of this study and helped in the carrying out this work . Also had role in analysing and writing up of the work

DR. K.K GOPINATHAN - Role in the planning of this study and helped in the carrying out this work

\section{Details of ethics approval :}

This study was approved by Institutional Ethics Committee of Edappal Hospitals Pvt.Ltd Approval Date$15 / 09 / 2018$

Reg no- BCR/815/INST/KL2016

IT no-06/18

Funding :- There was no funding for this submission .

\section{REFERENCES}

1 .Kady S, Gardosi J. Perinatal mortality and fetal growth restriction. Best Pract Res Clin Obstet Gynaecol 2004; 18: 397-410.

2. Jarvis S, Glinianaia SV, Torrioli MG, Platt MJ, Miceli M, Jouk PS, Johnson A, Hutton J, Hemming K, Hagberg G, Dolk H, Chalmers J. Cerebral palsy and intrauterine growth in single births: European collaborative study. Lancet 2003; 362: 1106-1111.

3. Barker DJ. Adult consequences of fetal growth restriction. Clin Obstet Gynecol 2006; 49: 270-283. 
4 .Schwarze A, Gembruch U, Krapp M, Katalinic A, Germer U, Axt-Fliedner R. Qualitative venous Doppler waveform analysis in preterm intrauterine growth-restricted fetuses with ARED flow in the umbilical artery - correlation with short-term outcome. Ultrasound Obstet Gynecol2005; 25: 573-579.

5 . Bilardo CM, Wolf H, Stigter RH, Ville Y, Baez E, Visser GH, Hecher K. Relationship between monitoring parameters and perinatal outcome in severe, early intrauterine growth restriction. Ultrasound Obstet Gynecol 2004; 23: 119-125.

6 . Fouron JC. The unrecognized physiological and clinical significance of the fetal aortic isthmus. Ultrasound Obstet Gynecol 2003; 22: 441-447.

7 . Eronen M, Kari A, Pesonen E, Kaaja R, Wallgren EI, Hallman M. Value of absent or retrograde enddiastolic flow in fetal aorta and umbilical artery as a predictor of perinatal outcome in pregnancy induces hypertension. Acta Pediatr 1993; 82: 919-924

8. Fouron JC, Gosselin J, Amiel-Tison C, Infante-Rivard C, Fouron C, Skoll A, Veilleux A. Correlation between prenatal velocity waveforms in the aortic isthmus and neurodevelopmental outcome between the ages of 2 and 4 years. Am J Obstet Gynecol 2001; 184: 630-636.

9 . Fouron JC, Gosselin J, Raboisson MJ, Lamoureux J, Tison CA, Fouron C, Hudon L. The relationship between an aortic isthmus blood flow velocity index and the postnatal neurodevelopmental status of fetuses with placental circulatory insufficiency. Am J Obstet Gynecol2005; 192: 497-503.

10. Figueras F, Benavides A, Del Rio M, Crispi F, Eixarch E, Martinez J, Hernandez-Andrade E, Gratacos E. Monitoring of fetuses with intrauterine growth restriction: longitudinal changes in ductus venosus and aortic isthmus flow. Ultrasound Obstet Gynecol 2009; 33: 39-43.

11. S. J. Gordijn*, i. M. Beune*, b. Thilaganathan+, a. Papageorghiou+,a. A. Baschat++, p. N. BakerSS, r. M. SilverP, k. Wynia** and w. Ganzevoort++ Consensus definition of fetal growth restriction: a Delphi procedure Ultrasound Obstet Gynecol 2016; 48: 333-339

12. Francesc Figueras Eduard Gratacos : Update on the Diagnosis and Classification of Fetal Growth Restriction and Proposal of a Stage-Based Management Protocol Fetal Diagn Ther 2014;36:86-98

13. M. Del rio, J.m. martinez, F. Figueras, M. Bennasar, A. Olivella, M. Palacio,

O. Coll, B. Puerto and E. Grataco' s Doppler assessment of the aortic isthmus and perinatal outcome in preterm fetuses with severe intrauterine growth restriction Ultrasound Obstet Gynecol 2008; 31: 41-47.

14..S. Hidar R. Zaafouri S. Bouguizane A. Chaieb Mr. Jerbi Mr. Bibi H. Khairi Prognostic contribution of Doppler of the fetal aortic isthmus in the management of iposterntrauterine growth retardation : Prospective longitudinal studyPrognostic value of fetal aortic isthmus Doppler waveform in growth restricted fetuses: prospective longitudinal study. Journal of Gynecology Obstetrics and Biology of Reproduction ,Volume 33, Issue 8 , December 2004, 745-752

15. Makikallio K, Jouppila P, Rasanen J. Retrograde net blood flow in the aortic isthmus in relation to human fetal arterial, and venous circulations. Ultrasound Obstet Gynecol 2002; 19: 147-152.

16. Monica Cruz-Lemini a Fatima Crispi a Tim Van Mieghem b Daniel Pedraza c

Rogelio Cruz-Martinez a Ruthy Acosta-Rojas a Francesc Figueras a auro Parra-Cordero c Jan Deprest b Eduard Gratacos

Risk of Perinatal Death in Early-Onset Intrauterine Growth Restriction according to Gestational Age and Cardiovascular Doppler Indices: A Multicenter Study Fetal Diagn Ther 2012;32:116-122.

\section{Hosted file}


Figures.docx available at https://authorea.com/users/301166/articles/430967-retrograde-flowin-aortic-isthmus-trigger-to-deliver-growth-restricted-fetuses-between-30-34-weeks-ofgestation 